\title{
THE DIOPHANTINE APPROXIMATION OF CERTAIN CONTINUED FRACTIONS
}

\author{
CHARLES F. OSGOOD
}

Abstract. Given a real number $\alpha$ defined by

$$
\frac{1}{\varphi(1)+} \frac{1}{\varphi(2)+} \cdots
$$

where $\varphi$ is a function from the natural numbers to the rational numbers larger than or equal to one which satisfies certain restrictions on the growth of the numerators and denominators of the numbers $\varphi(n)$, then a lower bound is found in terms of $\varphi$ for the diophantine approximation of $\alpha$.

We wish to consider the continued fraction

$$
\frac{1}{\varphi(1)+} \frac{1}{\varphi(2)+} \cdots
$$

where $\varphi(n)$ and an auxiliary function $\psi(n)$ satisfy certain conditions. Suppose that $\varphi(n)$ and $\psi(n)$ are functions defined from the positive integers to, respectively, the positive rationals and the positive integers such that

$$
\begin{gathered}
1 \leqq \varphi(j) \text { for } j=1,2, \cdots ; \\
\lim _{n \rightarrow \infty} \prod_{j=1}^{n} \varphi(j)=\infty, \text { and } \\
\lim _{n \rightarrow \infty} \sup (\log (2))(1+n / 2)\left(\log \left(\prod_{j=1}^{n-1} \varphi(j)\right)\right)^{-1}=\eta
\end{gathered}
$$

for some $0 \leqq \eta<+\infty$;

(c) $\quad 0 \leqq \lim _{n \rightarrow \infty} \sup (\log (\varphi(n+1)))\left(\log \left(\prod_{j=1}^{n} \varphi(j)\right)\right)^{-1}=\delta<+\infty$;

$$
\text { (e) } \quad \lim _{n \rightarrow \infty} \sup (\log (\psi(n)))\left(\log \left(\prod_{j=1}^{n} \varphi(j)\right)\right)^{-1}=\gamma<1 \text {. }
$$

Received by the editors July 19, 1971.

AMS 1970 subject classifications. Primary 10F20, $10 \mathrm{~F} 05$.

Key words and phrases. Diophantine approximation, continued fractions, rational partial quotients.

(c) American Mathematical Society 1972 
(Since each $\varphi(j)>0$ and $\sum_{j=1}^{\infty} \varphi(j) \geqq \sum_{j=1}^{\infty} 1=+\infty$, it follows immediately, from [1], that the continued fraction

$$
\frac{1}{\varphi(1)+} \frac{1}{\varphi(2)+} \cdots \frac{1}{\varphi(n)+} \cdots
$$

converges to a positive real number $\alpha$.)

THEOREM. For every $\varepsilon>0$, there exists a $c(\varepsilon)>0$ such that, for every pair of positive integers $p$ and $q$,

$$
\left|\alpha-p q^{-1}\right|>c(\varepsilon) q^{-(1+\theta+\varepsilon)}
$$

where $\theta=(1+\delta)(1+\eta+\gamma)(1-\gamma)^{-1}$.

EXAMPLES. We note that $\varphi(n)=n$ and $\psi(n)=1$ satisfy (a)-(e), as do $\varphi(n)=\left(t s^{-1}\right)^{n}$ and $\psi(n)=s^{n(n+1) / 2}$ for positive integers $s$ and $t$ with $t>s^{2}$. Also, with $s$ and $t$ as above, $\varphi(n)=p_{d}\left(\left(t s^{-1}\right)^{n}\right)$ and $\psi(n)=s^{n(n+1) d / 2}$ satisfy (a)-(e) where $p_{d}(x) \in Z^{+}[x]$ and $p_{d}(x)$ has degree $d \geqq 1$ in $x$. One may generalize this to see that, where $d_{j}, t_{j}$, and $s_{j}$, for $1 \leqq j \leqq m$, are positive integers satisfying

$$
\prod_{j=1}^{m} t_{j}^{d_{j}}>\left(\prod_{j=1}^{m} s_{j}^{d_{j}}\right)^{2}
$$

where $\varphi(n)=p_{d_{0}, \cdots, d_{m}}\left(n, q_{1}^{n}, \cdots, q_{m}^{n}\right)$ is a polynomial in $n, q_{1}^{n}=\left(t_{1} s_{1}^{-1}\right)^{n}, \cdots$, $q_{m}^{n}=\left(t_{m} s_{m}^{-1}\right)^{n}$ with positive integral coefficients and degrees $d_{0}, \cdots, d_{m}$, respectively, of the form a nonzero polynomial in $n$ times $q_{1}^{n d_{1}} \cdots q_{m}^{n d_{m}}$ plus terms of lower degree in some $q_{j}^{n}$, and where $\psi(n)=\prod_{j=1}^{m} s_{j}^{n(n+1) d_{j} / 2}$ then hypotheses (a)-(e) of the Theorem are satisfied also.

One may drop the assumption of positive coefficients in the $p_{d}(x)$ above. To see this, note first that the dominant term in each $\varphi(n)$ forces it to be of constant sign for large enough $n$. Then, for some positive integer $N$, the hypotheses of the Theorem are satisfied upon substituting

$$
\beta=\frac{1}{|\varphi(N)|+|\varphi(N+1)|+} \cdots \text { for } \alpha .
$$

The $(N+j)$ th convergent of the original continued fraction for $\alpha$ is given by $\left(A c_{j}+B\right)\left(C c_{j}+D\right)^{-1}$, for integers $A, B, C$, and $D$ with $\left.\right|_{C} ^{A}{ }_{D}^{R} \mid \neq 0$, where $c_{j}$ is the $j$ th convergent of the continued fraction above giving $\beta$ and $A C^{-1}$ and $B D^{-1}$ are, respectively, the $N$ th and $(N-1)$ st convergents of $\alpha$. Since $\beta$ is irrational (by (1)) eventually each $\left(A c_{j}+B\right)\left(C c_{j}+D\right)^{-1}$ is defined and, as $j \rightarrow \infty$, these convergents approach $(A \beta+B)(C \beta+D)^{-1}=$ $T(\beta)$, where $T(z)=(A z+B)(C z+D)^{-1}$. 
If $\left|\beta-T^{-1}\left(p q^{-1}\right)\right|<\left|\beta-D C^{-1}\right|$ we have, by the law of the mean, that $\left|T(\beta)-p q^{-1}\right|=\left|T(\beta)-T\left(T^{-1}\left(p q^{-1}\right)\right)\right|=\left|T^{\prime}(\xi)\right|\left|\beta-T^{-1}\left(p q^{-1}\right)\right|$ where $\xi$ is some point lying between $\beta$ and $T^{-1}\left(p q^{-1}\right)$. By the continuity of $T(z)$ at the irrational point $\beta$, we see the continuity of $T^{-1}(z)$ at $T(\beta)$ and this latter property implies that, by requiring $\left|T(\beta)-p q^{-1}\right|$ to be sufficiently small, we can guarantee $\left|\beta-T^{-1}\left(p q^{-1}\right)\right|<\left|\beta-D C^{-1}\right|$. Thus either

$$
\left|T(\beta)-p q^{-1}\right| \geqq K_{1}\left|\beta-T^{-1}\left(p q^{-1}\right)\right|
$$

for some constant $K_{1}>0$ independent of $p$ and $q$ or

$$
\left|T(\beta)-p q^{-1}\right|>K_{2}>0
$$

for some constant $K_{2}$ independent of $p$ and $q$. One may conclude, from (1) with $\alpha=\beta$ and the alternatives (i) and (ii) above, that (1) holds with $\alpha=T(\beta)$, for a $0<c_{1}(\varepsilon) \leqq c(\varepsilon)$ replacing $c(\varepsilon)$ in $(1)$; since, if $c_{1}(\varepsilon)<K_{2}$, case (ii) implies that (1) holds and case (i) says that $\left|T(\beta)-p q^{-1}\right| \geqq$ $K_{1}\left|\beta-T^{-1}\left(p q^{-1}\right)\right|$, which is larger than $c_{1}(\varepsilon) q^{-(1+\theta+\varepsilon)}$ for some $0<c_{1}(\varepsilon) \leqq$ $\min \left\{c(\varepsilon), K_{2}\right\}$ independent of $p$ and $q$.

In all of the above examples, $\delta$ was zero. To see a case in which $\delta>0$ set $\varphi(n)=(5 / 2)^{2^{n}}$ and $\psi(n)=\frac{1}{2}\left(2^{2^{n+1}}\right)$. One could generalize along the lines above and build up more complicated examples from this last example.

The author was led to consider the present problem after obtaining in [2], by different but related methods, a lower bound on the simultaneous diophantine approximation of the real number

$$
1+\frac{z q^{-1}}{1+} \frac{z q^{-2}}{1+} \cdots \frac{z q^{-n}}{1+}
$$

where $q$ denotes an integer, $z$ denotes a rational number, $|q|>1$, and $|z|>0$. (For $z=1$, the above number equals

$$
\prod_{n=0}^{\infty}\left(1-q^{-(5 n+2)}\right)\left(1-q^{-(5 n+1)}\right)^{-1}\left(1-q^{-(5 n+3)}\right)\left(1-q^{-(5 n+4)}\right)^{-1},
$$

as was shown by Ramanujan.) Since we may rewrite this real number as

$$
1+\frac{z}{q+} \frac{z}{q+} \frac{z}{q^{2}+} \frac{z}{q^{2}+} \cdots \frac{z}{q^{n}+} \frac{z}{q^{n}+} \frac{z}{q^{n+1}+} \cdots,
$$

it follows that the present theory applies at $z=1$. In each case, we obtain inequality (1) with $\theta=1$.

Proof of THE THeorem. Since $\sum_{j=2}^{\infty} p(j)=+\infty$ we see that the continued fraction with these partial quotients converges to a positive real 
number, $\alpha^{\prime}$. Now $\alpha=\left(\varphi(1)+\alpha^{\prime}\right)^{-1}$ so we have $\alpha<(\varphi(1))^{-1}$. Using induction set $a_{0}=1, a_{1}=\alpha<(\varphi(1))^{-1}, a_{2}=a_{1}\left(\left(a_{1} a_{0}^{-1}\right)^{-1}-\varphi(1)\right)<(\varphi(1) \varphi(2))^{-1}, \cdots$, $a_{n}=a_{n-1}\left(\left(a_{n-1} a_{n-2}^{-1}\right)^{-1}-\varphi(n-1)\right)<(\varphi(1) \cdots \varphi(n))^{-1}$. Note that no $a_{j}$ above is zero since each $a_{j}$ is the product of nonzero real numbers. Also for $n=2,3, \cdots$,

$$
a_{n}=-\varphi(n-1) a_{n-1}+a_{n-2} .
$$

Using (2) repeatedly, we may write each $\psi(n-1) a_{n}$ as a linear form $L_{n}=A_{n} a_{1}+B_{n} a_{0}$ for integers $A_{n}$ and $B_{n}$ satisfying easily calculable upper bounds on their absolute values. We find that

$$
\left|B_{n}\right| \leqq \sum_{0 \leqq k \leqq n / 2}\left(\begin{array}{c}
n-k \\
k
\end{array}\right)\left(\prod_{j=1}^{n-1} \varphi(j)\right) \psi(n-1),
$$

where $\left(\begin{array}{c}n-k \\ k\end{array}\right)<2^{n-k}$. Thus $B_{n}<2^{1+n / 2}\left(\prod_{j=1}^{n-1} \varphi(j)\right) \psi(n-1)$. Similarly,

$$
\begin{aligned}
\left|A_{n}\right| & \leqq \sum_{0 \leqq k \leqq n / 2}\left(\begin{array}{c}
n-1-k \\
k
\end{array}\right)\left(\prod_{j=1}^{n-1} \varphi(j)\right) \psi(n-1) \\
& <2^{1+n / 2}\left(\prod_{j=1}^{n-1} \varphi(j)\right) \psi(n-1) .
\end{aligned}
$$

Using hypotheses (b) and (e), for every $\varepsilon>0$,

$$
\max \left\{\left|A_{n}\right|,\left|B_{n}\right|\right\}<\left(\prod_{j=1}^{n-1} \varphi(j)\right)^{(1+\eta+\gamma+\varepsilon)}
$$

if $n$ is sufficiently large. Under these same conditions we have, using (c), that

$$
\max \left\{\left|A_{n}\right|,\left|A_{n+1}\right|,\left|B_{n}\right|,\left|B_{n+1}\right|\right\}<\left(\prod_{j=1}^{n} \varphi(j)\right)^{(1+\eta+\gamma+\varepsilon)} .
$$

Also, if $n$ is sufficiently large,

$$
\max \left\{\left|L_{n}\right|,\left|L_{n+1}\right|\right\} \leqq\left(\prod_{j=1}^{n} \varphi(j)\right)^{-(1-\gamma)+\varepsilon}
$$

From (2), we may write

$$
\left(\begin{array}{c}
a_{n} \\
a_{n+1}
\end{array}\right)=\left(\begin{array}{cc}
0 & 1 \\
1 & -\phi(n)
\end{array}\right)\left(\begin{array}{c}
a_{n-1} \\
a_{n}
\end{array}\right)
$$

The entries of

$$
\left(\begin{array}{cc}
0 & 1 \\
1 & -\varphi(n)
\end{array}\right)\left(\begin{array}{cc}
0 & 1 \\
1 & -\varphi(n-1)
\end{array}\right) \cdots\left(\begin{array}{cc}
0 & 1 \\
1 & -\varphi(1)
\end{array}\right)
$$


must be proportional to those of

$$
\left(\begin{array}{cc}
A_{n} & B_{n} \\
A_{n+1} & B_{n+1}
\end{array}\right)
$$

therefore

$$
\left|\begin{array}{cc}
A_{n} & B_{n} \\
A_{n+1} & B_{n+1}
\end{array}\right| \neq 0 .
$$

For each real $1 \leqq x<\infty$ set

$$
f(x)=\left(\left(\prod_{j=1}^{[x]} \varphi(j)\right)(\varphi([x]+1))^{x-[x]}\right)^{\theta_{1}}
$$

where $[x]$ denotes the greatest integer function and $\theta_{1}=(1+\delta)(1+\eta+\gamma)=$ $(1-\gamma) \theta$. Note that $f(x)$ is monotone increasing, and that $f(x)$ takes the nonnegative reals onto $[\varphi(1),+\infty)$, since $\prod_{j=1}^{\infty} \varphi(j)=\infty$. For $n=1, \cdots$ set

$$
\Delta_{n}=\left(\begin{array}{cc}
A_{n} & B_{n} \\
A_{n+1} & B_{n+1}
\end{array}\right) \quad \text { and } \quad V=\left(\begin{array}{l}
1 \\
\alpha
\end{array}\right) .
$$

Let $\|$ matrix $\|$ denote the maximum of the absolute values of its entries. Then given $0<\varepsilon<1$, if $n_{1}$ is sufficiently large we have, for all $n \geqq n_{1}$,

(i) $\left\|\Delta_{n}\right\| \leqq(f(n))^{1+\varepsilon / 5}$, from (3),

(ii) $\left\|\Delta_{n} V\right\| \leqq(f(n))^{-\theta_{2}^{-1}(1-\varepsilon / 5)}$, from (4), where $\theta_{2}=(1+\eta+\gamma)(1-\gamma)^{-1}$, and

(iii) $f(n) \leqq(f(n-1))^{(1+\delta)(1+\varepsilon / 5)}$ from (c).

We shall next state a lemma from which we will be able to conclude the present Theorem. Then we shall conclude this paper with a proof of the Lemma.

Suppose that for some positive integer $t$ we have a sequence $\Delta_{m}$ of $t$ by $t$ nonsingular matrices over the Gaussian integers and a $t$ by 1 matrix $V \neq 0$ with complex entries. Let $f(n)$ be a monotone increasing function from $[1,+\infty)$ onto $[c,+\infty)$ for some $c \geqq 1$. Let $0<\varepsilon<+\infty, 0<r<+\infty$, and $(1+\varepsilon / r)^{2}(1-\varepsilon / r)^{-1}<1+\varepsilon$. Suppose that, for all nonnegative integers $n \geqq n_{1} \geqq 1$,

(i) $\left\|\Delta_{n}\right\| \leqq(f(n))^{1+\varepsilon / r}$,

(ii) $\left\|\Delta_{n} V\right\| \leqq(f(n))^{-\Lambda(1-\varepsilon / r)}$ for some $\Lambda>0$, and

(iii) $f(n) \leqq(f(n-1))^{(1+\delta)(1+\varepsilon / r)}$.

Let $q$ denote a nonzero Gaussian integer and $P$ denote a general $t$ by 1 matrix of Gaussian integers with not all entries zero.

LEMMA. If $|q|>\frac{1}{2}\left(f\left(n_{1}\right)\right)^{\Lambda(1-\varepsilon / r)}$ then $\left\|V-P q^{-1}\right\| \geqq t^{-1}|2 q|^{-(1+(1+\varepsilon)(1+\delta) / \Lambda)}$. 
Note that if $0<\varepsilon<1$ then $(1+\varepsilon / 5)^{2}(1-\varepsilon / 5)^{-1}<(1+\varepsilon / 5)^{2}(1+\varepsilon / 4)<$ $(1+\varepsilon / 4)^{3}<1+61(64)^{-1} \varepsilon<1+\varepsilon$. One may then apply the Lemma to our present situation with $\Lambda=\theta_{2}^{-1}=(1-\gamma)(1+\eta+\gamma)^{-1}=(1+\delta) \theta^{-1}$. Thus we see that

$$
\left\|V-P q^{-1}\right\| \geqq \frac{1}{2}|2 q|^{-(1+(1+\varepsilon) \theta)},
$$

where $V=\left(\begin{array}{l}1 \\ \alpha\end{array}\right)$. Setting $P=\left(\begin{array}{l}q \\ p\end{array}\right)$, where $p$ is an arbitrary nonnegative integer, and letting $q$ be an arbitrary positive integer, we obtain

$$
\left|\alpha-p q^{-1}\right|>q^{-(1+(1+\varepsilon) \theta)}
$$

if $q$ is sufficiently large. Since (6) would be impossible if $\alpha$ were rational, we see that there must exist a $c(\varepsilon)>0$ such that $\left|\alpha-p q^{-1}\right|>c(\varepsilon) q^{-(1+\theta+\varepsilon)}$ if $q \geqq 1$. This proves our Theorem, assuming the Lemma.

Proof of the Lemma. For each nonnegative integer $n$,

$$
\left\|\Delta_{n}\left(V-P q^{-1}\right)\right\| \geqq\left\|\Delta_{n} P q^{-1}\right\|-\left\|\Delta_{n} V\right\| .
$$

Then $\left\|\Delta_{n} P q^{-1}\right\| \geqq|q|^{-1}$, and

$$
\left\|\Delta_{n}\left(V-P q^{-1}\right)\right\| \geqq|q|^{-1}-\left\|\Delta_{n} V\right\|,
$$

since each entry in $P$ and $\Delta_{n}$ is a Gaussian integer, $P \neq 0$, and each $\Delta_{n}$ is nonsingular. We now choose $n$ to be the first integer such that $|2 q|<$ $(f(n))^{\Lambda(1-\varepsilon / r)}$. Since $\left(f\left(n_{1}\right)\right)^{\Lambda(1-\varepsilon / r)}<|2 q|<(f(n))^{\Lambda(1-\varepsilon / r)}$ and $f(n)$ is monotone increasing we have $n>n_{1}$.

Therefore, we may use (ii) with (7) to conclude that

$$
\left\|\Delta_{n}\left(V-P q^{-1}\right)\right\| \geqq|q|^{-1}-(f(n))^{-\Lambda(1-\varepsilon / r)} .
$$

Since $|2 q|^{-1}>(f(n))^{-\Lambda(1-\varepsilon / r)}$ we have

$$
\left\|\Delta_{n}\left(V \cdot-P q^{-1}\right)\right\| \geqq|2 q|^{-1} .
$$

From our choice of $n$ and from hypothesis (iii), we see that

$$
\begin{aligned}
\exp \left((\log |2 q|) \Lambda^{-1}(1+\varepsilon / r)^{2}(1-\varepsilon / r)^{-1}(1+\delta)\right) \\
\geqq(f(n-1))^{(1+\varepsilon / r)^{2}(1+\delta)} \geqq(f(n))^{(1+\varepsilon / r)} .
\end{aligned}
$$

Since $(1+\varepsilon / r)^{2}(1-\varepsilon / r)^{-1}<1+\varepsilon$, we see that

$$
(f(n))^{1+\varepsilon / r} \leqq|2 q|^{(1+z)(1+\delta) \Lambda^{-1}} .
$$

From (8), hypothesis (i), and (9), we conclude that

$$
\begin{aligned}
\left\|V-P q^{-1}\right\| & \geqq t^{-1}|2 q|^{-1}\left\|\Delta_{n}\right\|^{-1} \geqq t^{-1}|2 q|^{-1}(f(n))^{-(1+\varepsilon / \tau)} \\
& \geqq t^{-1}|2 q|^{-(1+(1+\varepsilon)(1+\delta) \Lambda} .
\end{aligned}
$$

This proves the Lemma. 
The author wishes to thank the referee for pointing out a computational error in the original manuscript which when corrected enabled the author to strengthen his result.

\section{REFERENCES}

1. A. Ja. Hinčin, Continued fractions, GITTL, Moscow, 1961; English transl., Univ. of Chicago Press, Chicago, III., 1964. MR 13, 444; MR 28 \#5038.

2. C. F. Osgood, On the Diophantine approximation of values of functions satisfying certain linear q-difference equations, J. Number Theory 3 (1971), 159-177.

Mathematics Research Center, Naval Research laboratory, Washington, D.C. 20390 